\title{
Suitability of Visual Modelling Languages for Modelling Tangible User Interface Applications
}

\author{
Eric Tobias, Eric Ras \\ Service Science \& Innovation Department \\ Public Research Centre Henri Tudor \\ J.F. Kennedy av. 29, L-1855 Luxembourg, Luxembourg \\ Email: $\{$ eric.tobias, eric.ras\}@ tudor.lu
}

\author{
Nuno Amálio \\ Faculty of Science Technology and Communication \\ University of Luxembourg, Luxembourg \\ rue Richard Coudenhove-Kalergi 6, L-1359 Luxembourg \\ Email: nuno.amalio@uni.lu
}

\begin{abstract}
This paper compares the suitability of visual modelling languages for describing tangible user interface (TUI) applications. After gathering different approaches, we have selected three languages for our comparative study: the visual object constraint language (VOCL), augmented constraint diagrams (ACD), and the visual contract language (VCL). A weighted evaluation based on multiple quality criteria led to the conclusion that VCL is best suited to model TUI applications.
\end{abstract}

Index Terms-Visual Languages; Model Driven Development; Comparative Study; VOCL; Constraint Diagrams; VCL

\section{INTRODUCTION}

There is considerable hype surrounding domain-specific languages (DSLs), which is an attractive idea that has proved useful for certain problems. However, this hype can often be misleading: one may be tempted to invent a new DSL rather than trying to find a suitable general-purpose language.

This paper evaluates the suitability of general-purpose visual modelling languages (VMLs) to the design of tangible user interface (TUI) [1] applications. This is part of our research work on modelling a TUI application. TUIs manipulate a digital world using physical devices and projections to take advantage of known metaphors of the physical world. Our TUI application consists of an environment for building Business Process Modeling Notation 2.0 (BPMN2) diagrams.

\section{INCLUSION AND SELECTION CRITERIA}

We must determine what to compare and how to compare. The how is addressed in section III. The what or the suitability was determined by the following criteria:

- The languages need to be visual. This is an appropriate medium for the scope of TUI development. The language must be able do describe visually all properties of interest. Another strong criteria is the need for a modelling rather than a programming language.

- The study only considers general-purpose languages. A feature of TUIs is their strong interaction with the real world that often needs to be modelled. We therefore need languages that are capable of dealing with this diversity and that are capable of describing modelling requirements across many fields, yet specialised languages might only be suited for a specific task. Furthermore, a more general and abstract nature enables the language to use metaphors

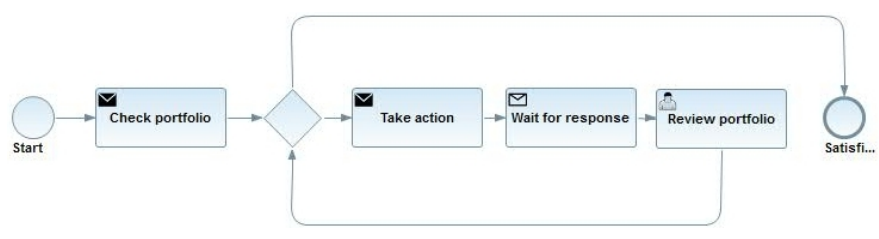

Fig. 1. BPMN2 scenario.

and abstractions to draw upon existing language schemata and improve language learning [2]. As noted by Schema Theory [3], the ability to understand a new domain can be improved by reusing familiar concepts in already existing schemata.

\section{STUDY}

From the thirty one gathered resources, only roughly half remained after pre-selection. Those were then further grouped and distilled to identify three major contestants:

- UML2 with the visual object constraint language (VOCL) [4].

- Augmented constraint diagrams (ACD) [5].

- The visual contract language (VCL) [6].

To perform the comparison, a simple BPMN2 scenario was devised (Fig. 1) and the relevant requirements extracted. These requirements were then modelled with each VML to gather data on their performance. The scenario and each modelling endeavour were undertaken by one of the authors to counteract the effect of familiarity, skill and understanding of the task on the result. It is to note that the author doing the modelling had some experience in all chosen VMLs apart from ACD.

The measurements categories collected after each modelling step are summarised in Table I. Each measure was attributed points according to Table II. Further details on measurements categories and scoring scheme can be found in the accompanying technical report [7].

\section{REsults}

The study's scores were marked as by the scoring scheme and subsequently weighted. The weighting, being the result

\footnotetext{
${ }^{1}$ Where $\mathrm{X}$ is either structural requirements, behavioural requirements or constraints.
} 


\begin{tabular}{|l|l|}
\hline \multirow{3}{*}{ Tool support } & $\begin{array}{l}\text { Availability } \\
\text { Maintained } \\
\text { Latest version } \\
\text { Branch }\end{array}$ \\
\hline \multirow{3}{*}{ Semantics \& Transformation } & $\begin{array}{l}\text { Formally defined } \\
\text { Transformability }\end{array}$ \\
\hline \multirow{5}{*}{ Expressivity } & $\begin{array}{l}\text { \# [ X ] } \\
\text { \# [ X ] satisfied } \\
\text { \# requirements partially satisfied } \\
\text { \# unsatisfied requirements } \\
\text { Ratio }\end{array}$ \\
\hline \multirow{5}{*}{ Usability } & Naming conventions \\
& Naming fit \\
& Documentation \\
& Tutorial \\
& Hands-on-tutorial \\
& Primitive mutability \\
& Live suggestions \\
\hline \multirow{5}{*}{ Error Checking } & Time \\
& Syntax highlighting \\
& Degree \\
& Error correction suggestion \\
& Debugging possible \\
\hline Validation \& Verification & Modularity \\
& Verification scheme \\
\hline
\end{tabular}

TABLE I

MEASUREMENT CATEGORIES

\begin{tabular}{|c|l|}
\hline 10 & Desirable \\
\hline 5 & Manageable \\
\hline 2 & Acceptable \\
\hline-5 & Undesirable \\
\hline$-\infty$ & Disqualification criterion \\
\hline
\end{tabular}

TABLE II

SCORING SCHEME

of a questionnaire sent out to a test sample of practitioners proved to not affect the outcome significantly. See [7] for further details. The evaluation scores for this small study can be seen in Table III. The results show that VCL surpasses ACD due to the latter's non-existent tool support, which makes error checking tedious. The combination of UML2 \& VOCL looses ground on tool support, when compared to VCL, and formalism, due to lack of formality for some parts of UML2.

\section{CONCLUSIONS}

The study presented here is a preliminary evaluation done with the purpose of choosing a VML for modelling a TUI application. To the authors knowledge, this is the first study comparing this particular set of VMLs. Although the evaluation has been done using a small case study, the results are interesting giving a good overall picture on the state of the art in visual modelling approaches. The fact that three generalpurpose modelling languages are, at first sight, suited to model TUIs is encouraging. For this small case study, all evaluated VMLs were capable of describing the requirements. Some VMLs have seen little development since their inception, after providing a proof of concept. It was VCL, the most recently

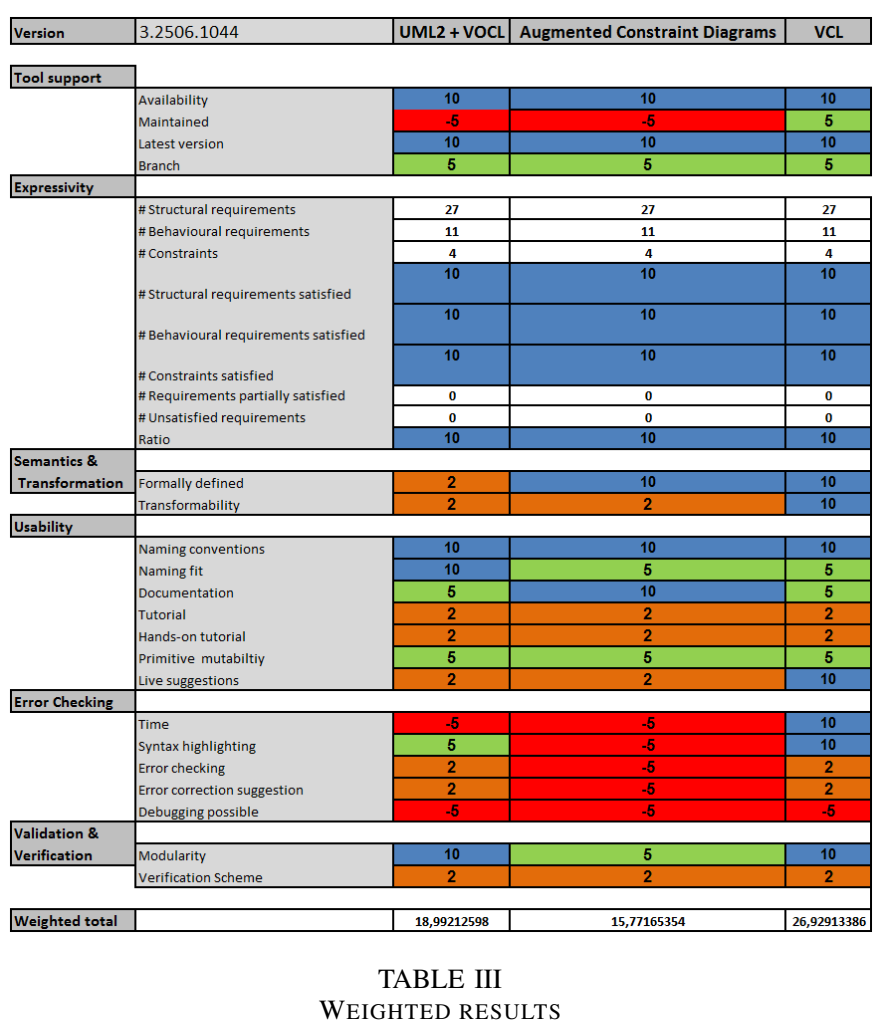

developed VML and hence still actively being support and featuring modern model-driven facilities, that showed to provide an advantage over the other contestants. This advantage may, however, vane with the conclusion of the project. A future goal of being able to visually model applications directly using VCL in a collaborative TUI environment is not unreachable if VCL continues to be supported.

\section{REFERENCES}

[1] O. Shaer and E. Hornecker, "Tangible user interfaces: Past, present, and future directions," Foundations and trends in Human Computer Interaction, vol. 3, no. 1-2, pp. 1-137, 2009.

[2] F. Boers, "Metaphor awareness and vocabulary retention," Applied linguistics, vol. 21, no. 4, pp. 553-571, Dec. 2000.

[3] S. A. Widmayer, "Schema theory: An introduction," Retrieved April, 2005.

[4] K. Ehrig and J. Winkelmann, "Model transformation from visual OCL to OCL using graph transformation," ENTCS, vol. 152, pp. 23-37, 2006.

[5] J. Howse, S. Schuman, G. Stapleton, and I. Oliver, "Diagrammatic Formal Specification of a Configuration Control Platform," Electronic Notes in Theoretical Computer Science, vol. 259, pp. 87-104, Dec. 2009.

[6] N. Amálio and P. Kelsen, "Modular design by contract visually and formally using VCL," in VL/HCC 2010, 2010.

[7] E. Tobias, E. Ras, and N. Amálio, "VML Usability for Modelling TUI Scenarios - A Comparative Study," University of Luxembourg, LASSY, Tech. Rep. TR-LASSY-12-06, 2012, available at http://vcl.gforge.uni.lu/ doc/VMLCaseStudy.pdf. 